\title{
Búsqueda y evaluación de información: dos competencias necesarias en el contexto de las fake news
}

\author{
Alonso Varela, Lucía; Saraiva Cruz, Ignacio \\ Búsqueda y evaluación de información: dos competencias necesarias en el contexto de las fake news \\ Palabra Clave (La Plata), vol. 9, núm. 2, 2020 \\ Universidad Nacional de La Plata, Argentina \\ Disponible en: http://www.redalyc.org/articulo.oa?id=350562513003 \\ DOI: https://doi.org/10.24215/18539912e090
}




\section{Búsqueda y evaluación de información: dos competencias necesarias en el contexto de las fake news}

Information search and information evaluation: two necessary competencies in fake news context.

Lucía Alonso Varela

Universidad de la República. Facultad de Información y

Comunicación, Uruguay

lucia.alonso@fic.edu.uy

Ignacio Saraiva Cruz

Universidad de la República. Facultad de Información y

Comunicación, Uruguay

ignacio.saraiva@fic.edu.uy
DOI: https://doi.org/10.24215/18539912e 090

Redalyc: http://www.redalyc.org/articulo.oa? id $=350562513003$

Recepción: 26 Mayo 2019

Aprobación: 27 Diciembre 2019

\section{RESUMEN:}

En la sociedad de la información, la creación y difusión de información ha tenido un crecimiento exponencial, lo que representa un reto para los individuos a la hora de acceder a información confiable. A partir de una revisión bibliográfica, se presentan y discuten tres conceptos clave: búsqueda de información, evaluación de información y fake news. Se conceptualizan los dos primeros como competencias indispensables para enfrentar a las fake news. Asimismo, se exponen algunas definiciones e implicancias que estas competencias poseen para formar a los individuos en el manejo de la información. El fenómeno de las fake news demanda la adquisición de estas competencias, centrales para discernir entre la información confiable y la que no lo es. Por último, se posiciona al profesional de la información como actor de relevancia en el desarrollo de competencias en información, y a la ciencia de la información como parte del debate de esta temática, por integrar el mundo informacional y comunicacional.

Palabras clave: Búsqueda de información, Evaluación de información, Competencias en información, Alfabetización en información, Noticias falsas.

\section{ABstract:}

In the information society, the creation and dissemination of information has had an exponential growth, which represents a challenge for individuals when it comes to accessing reliable information. From a bibliographic review, three key concepts are presented and discussed: information search, information evaluation and fake news. The first two are conceptualized as indispensable competencies to face fake news. Likewise, some definitions and implications that these competencies have to train individuals in the handling of information are exposed. The phenomenon of fake news demands the acquisition of these competencies, central to discern between reliable and non-reliable information. Finally, the information professional is positioned as a relevant actor in the development of information competencies, and information science as part of the debate on this subject, for integrating the informational and communicational world.

KEYWORDS: Information search, Information evaluation, Information competencies, Information literacy, Fake news.

\section{INTRODUCCión}

En la actualidad nos encontramos situados en la llamada sociedad de la información; sociedad en la que se persigue la adquisición, almacenamiento, procesamiento, evaluación, transmisión, difusión y diseminación de la información en pro de la elaboración de conocimiento y la satisfacción de necesidades individuales y colectivas. Caracterizada por la explosión de información, y el crecimiento notorio del almacenamiento y uso datos, con la particularidad de poseer una multiplicidad de fuentes de información y de canales de transmisión, resulta de todo ello el caos informacional que define a dicha sociedad. A esa realidad se 
suma el impacto de las tecnologías de la información y la comunicación, herramientas para el manejo de la información.

En el marco de esta sociedad, y como consecuencia de la misma, se considera que es necesario que los sujetos posean las competencias de buscar, evaluar y seleccionar las diversas fuentes de información que nos proporciona el universo informacional, en orden de discernir cuales son pertinentes y de calidad y cuáles no lo son. La adquisición de competencias en información como resultado del proceso de alfabetización en información, resulta imprescindible para el desarrollo individual y de la sociedad en su conjunto.

En este sentido, el objetivo del presente artículo es analizar el fenómeno de las fake news y su vínculo con las competencias en información. Para lograrlo, se recurre a un trabajo de revisión bibliográfica, a partir del cual, se presentan y discuten los conceptos de búsqueda de información, evaluación de información y fake news. Se abordan algunas de las implicancias que estas competencias poseen a la hora de formar a los individuos en el manejo de la información. Esto se torna de gran relevancia en el contexto de circulación y difusión de las fake news, las cuales, a partir de información falsa, intentan desinformar a las personas. Como consecuencia, las competencias de búsqueda y evaluación de la información ${ }^{1}$ toman una nueva dimensión y se vuelven indispensables.

\section{COMPETENCIAS PARA EL MANEJO DE INFORMACIÓN}

Con relación al concepto de competencias informacionales o competencias en el manejo de información, se puede apreciar que existen varias acepciones. En este sentido, Ruiz y Chapman (2017) consideran que es necesario establecer de manera clara la diferencia entre la noción de competencia y el resto de los procesos dentro de la alfabetización en información, ya que suelen prestarse a confusión. Al respecto, reparan en que las competencias en información se determinan por tres elementos constitutivos, los cuales son: los conocimientos, las habilidades y las actitudes.

En virtud de ello, especificar y analizar estas competencias permitirá comprender su relevancia a la hora de que los individuos se desempeñen en los diversos contextos y canales de comunicación de información. American Association of School Librarians \& Association for Educational Communications And Technology (1998, p. 2) establecen que la competencia informativa es:

la habilidad de reconocer una necesidad de información y la capacidad de identificar, localizar, evaluar, organizar, comunicar y utilizar la información de forma efectiva, tanto para la resolución de problemas como para el aprendizaje a lo largo de la vida (traducción propia).

En la misma línea, Mata, Silva Casarin y Marzal (2016, p. 296) entienden que "se comprende que la competencia informacional ocurre por medio de un proceso de ensayo-aprendizaje, propiciando la formación de conocimientos, habilidades y actitudes sobre el universo informacional en los individuos [...] (traducción propia). A su vez, al referirse a lo que generan en los sujetos las competencias en información, indican que:

A partir de la apropiación de dicho conocimiento, el individuo puede aplicarlo en el contexto en el que se inserta, desde realizar tareas simples hasta las más complejas, como la búsqueda de información para la resolución de problemas personales, el desarrollo de trabajos escolares / académicos, la evaluación información para verificar su autenticidad y confiabilidad en referencia a diversas situaciones [...] le permite utilizar la información para actuar en el entorno en el que se inserta de manera ética (Mata, Silva Casarin y Marzal, 2016, p. 297, traducción propia).

Sumado a ello, Ceretta Soria, Canzani Cuello y Cabrera Castiglioni (2016) consideran que, las competencias en el manejo o tratamiento de la información son aquellas involucradas en el proceso que engloba la búsqueda, acceso, selección, evaluación y uso de la información.

Así, dichas competencias pueden entenderse como el conjunto de habilidades, conocimientos, actitudes y capacidades que le permiten al individuo llevar un óptimo manejo de la información; identificando necesidades informativas, buscando y procesando los recursos informativos pertinentes para convertir dicha 
información en conocimientos, los cuales le sean útiles para solucionar las diversas problemáticas que se le presenten. Comprendidas de esta forma, se puede considerar que se trata de competencias básicas que los sujetos deben poseer para su desarrollo. Estas competencias se recogen en varias normas de alfabetización en información. Según Fernández-Ramos (2014, p. 119):

estas normas o estándares se hacen eco de la necesidad, especialmente en la sociedad de la información y del conocimiento, de que la gente sea capaz de identificar sus necesidades de información, de saber buscarla, evaluarla, organizarla y utilizarla adecuadamente.

La generación de competencias en información proporciona potencialidades en el individuo que propician su desarrollo individual y colectivo, facilitando la gestión autónoma de información y la construcción de conocimiento (Batista Rojas, Hernández Rodríguez, Hernández López y González Cedeño, 2011; Elisondo y Donolo, 2014).

De esta manera, las competencias adquiridas como resultado del proceso de alfabetización en información, son herramientas indispensables para optimizar su manejo y tratamiento. Es interesante remarcar el rol que tienen los profesionales de la información en la actualidad. Éstos se posicionan como agentes educativos, ya que una de sus funciones primordiales es la de formar a los individuos para vincularse con el mundo informacional.

\subsection{Búsqueda de información}

Establecido brevemente el concepto de competencias en el manejo de la información, es claro que, uno de los pilares fundamentales en su desarrollo es la búsqueda de información ${ }^{2}$ y, es por esto que, representa una de las categorías analíticas en el presente trabajo.

El individuo que se encuentra frente a una necesidad de información, deberá llevar a cabo una búsqueda adecuada para encontrar su respuesta. La misma debe interpretarse como un proceso en el cual inciden diversos factores y se encuentra directamente relacionado con el contexto del individuo que la realiza, así como, con su necesidad informacional. Sobre esta base, las etapas claves que definen a la búsqueda de información son: analizar y comprender la problemática; identificar en donde se realizará la búsqueda; interactuar y realizar la búsqueda en el lugar seleccionado; identificar la pertinencia de la información obtenida en base a la necesidad de información; organizar la información que responde a la problemática (Argudo y Pons, 2012; Cid Leal y Perpinya Morera, 2013).

Este proceso es central en el marco de las competencias en información, por lo que resulta necesario que se le otorgue la relevancia adecuada. El profesional de la información debe desarrollar de la competencia vinculada con la búsqueda de información puesto que, en la actualidad, se encuentra presente en todos los ámbitos de la vida cotidiana y es transversal a los procesos educativos e informativos de los individuos.

Comprender cómo encontrar y buscar información es un pilar fundamental para el adecuado desenvolvimiento en la sociedad. La búsqueda puede ser realizada en diversos medios y canales de transmisión de información, desde internet a un catálogo manual. Como consecuencia, los individuos necesitan distintos tipos de fuentes de información, según el contexto y la respuesta que buscan (Mata, Silva Casarin, Marzal, 2016). En este sentido, Budd y Llyod (2014, p. 4), al hablar sobre alfabetización en información, pero que repercute en el abordaje de la búsqueda de información, aclaran que:

[...] se reconoce que la información existe en cada comunidad y funciona al proporcionar una forma de coherencia (lo que "todos saben" en una comunidad determinada). Cuando cruzamos los límites culturales, ya sea metafóricamente o literalmente, la información cambia de forma y significado [...] En buenos sistemas y en buenas interacciones, la información se traduce entre culturas y contextos. Esta actividad de traducción podría verse como una de las funciones centrales de la alfabetización informacional como concepto sociocultural y uno de los principios clave para el diseño exitoso de un sistema de información (traducción propia). 
De esto se desprende que la alfabetización en información y la búsqueda de información no están exentas de un contexto cultural. La búsqueda debe ser vista por el individuo como una primera herramienta a la hora de satisfacer sus necesidades de información. Mientras que la alfabetización debe ser un proceso que permita reconocer y contextualizar la información imprescindible para dar respuesta a una demanda de información. La disponibilidad y difusión de información se encuentra muy extendida, por lo que, al realizar una búsqueda se debe precisar qué tipo de información es la que se necesita, para luego establecer dónde se debe buscar.

En virtud de ello, se puede considerar a la búsqueda de información como una competencia básica para el manejo de la misma; y un proceso que implica varias etapas y estrategias, con el fin de satisfacer una necesidad. Así, el individuo debe traducir la necesidad de información y transformarla en una consulta que le permitirá acceder a diversas fuentes (Ladrón de Guevara, 2017; López Flamarique, 2017; Piwowarski y Blanco González, 2011). Tal como lo mencionan Budd y Llyod (2014) el proceso de alfabetización y el de búsqueda están influenciados por aspectos socioculturales que modifican las estrategias y formas en que estas necesidades se presentan.

Para que los individuos puedan realizar un uso adecuado de la información, es necesario que se encuentren alfabetizados en su manejo. Si nos referimos específicamente al uso y manejo en los entornos web, es imprescindible poseer competencias en la búsqueda ya que es el primer paso dentro de las estrategias para dar respuesta a las necesidades de información.

En este sentido, recientemente Cocklar, Yaman y Yurdakul (2017) llevaron a cabo un estudio sobre estudiantes de pregrado en orden de analizar las competencias de búsqueda de información en los entornos web, la relación entre la natividad digital y la alfabetización en información. En el mismo, consideran que, aquellos usuarios que poseen un manejo de la información escaso corren riesgo de no poder detectar y validar la fiabilidad y veracidad de los contenidos, ni localizar su autoría, elementos clave para poder utilizar y explotar adecuadamente la información en línea.

Los autores concluyen que el proceso de alfabetización en información posee una correlación de alto nivel con saber buscar información en internet, mientras que, la natividad digital posee una correlación de bajo nivel con la búsqueda. En consecuencia, estiman que aquellos estudiantes que no han adquirido competencias en información son más propensos a aceptar información falsa, manipulada y parcializada, lo que no les permite alcanzar un pleno desarrollo tanto en el ámbito académico como en el social.

De esta manera, si se espera que los estudiantes se beneficien con la información Recuperado de la web, es necesario que se encuentren alfabetizados informacionalmente ya que las estrategias de búsqueda son elementos fundamentales para este proceso. (Cocklar, Yaman y Yurdakul, 2017).

Sobre esta línea, Odede y Zawedde (2018) coinciden que el adecuado uso de los recursos de información electrónica por parte de los usuarios se encuentra íntimamente relacionado con el desarrollo de la alfabetización y las competencias en información, en donde la búsqueda es un elemento central para guiarse en el mundo informacional.

La búsqueda de información es, por tanto, un proceso complejo y dinámico que se encuentra influenciado por varios factores. Requiere de la adquisición de herramientas competenciales para encontrar la información específica requerida. Poseer las competencias de búsqueda en un contexto en donde los individuos están constantemente interactuando con la información en la web es de gran relevancia. Sumado a esto, en la actualidad, donde la información falsa y las fake news están cada vez más presentes, se torna sumamente necesario que los individuos manejen buenas estrategias para la búsqueda de información.

\subsection{Evaluación de información}

Como segunda categoría analítica para la discusión que aquí se presenta, se toma la evaluación de información, otro de los pilares indispensables para el correcto manejo de información. Evaluar, para posteriormente seleccionar recursos de información de calidad, es cada vez más necesario. A continuación, se 
definen los conceptos evaluación, selección y calidad, por entender que son los elementos clave del proceso de evaluación de recursos de información.

Según Salvador Oliván y Angós Ullate (2001, p. 107), la evaluación se define como:

la aplicación de un conjunto de métodos y técnicas de investigación que permiten obtener información fiable, para la toma de decisiones en las tareas de desarrollo, mantenimiento y gestión de los sistemas y servicios de información... supone la aplicación de métodos y criterios científicos con el fin de mejorar la toma de decisiones.

Resulta pertinente aclarar que selección es un término proveniente etimológicamente del latín seligere que refiere a "escoger poniendo a parte" (Corominas, 2012, p. 204); es decir, alude a un proceso de decisión que el individuo lleva adelante y que tiene por resultado elegir y descartar elementos frente a un conjunto determinado.

Vinculado a ella, cabe mencionar el concepto de calidad, sobre el que Voutssas-Márquez (2017, p. 141), luego de examinar diversos requisitos utilizados para establecer la calidad de la información, considera que se encuentra íntimamente relacionada con los requerimientos de los individuos y con la capacidad que posee de satisfacer sus necesidades.

Así, se puede establecer que la evaluación de información es el proceso que tiene como objetivo seleccionar, es decir, escoger algunas fuentes de información por sobre otras, a partir de la calidad de la información que contienen como criterio de selección.

Es necesario además que los individuos posean habilidades que les permitan discernir si la información que encuentran les es útil para cada problema de información que deban resolver (Argudo, Pons, 2012).

De esta modo, la calidad de una fuente de información está íntimamente relacionada con la necesidad informacional de los individuos. La evaluación de la información posee elementos subjetivos, ya que la misma viene dada por la capacidad de dar respuesta a la consulta realizada en la etapa de búsqueda de información (Delis Alfonso, 2016). También es necesario comprender que existen un conjunto de particularidades deseables al momento de realizar la evaluación. Sobre esta línea, se puede apuntar que: [...] aunque no es posible establecer reglas rigurosas de confiabilidad para los contenidos [...] [los usuarios] debe[n] disponer de una serie de directrices que les permitan evaluar la información para determinar su calidad (Giffoni Dutra, Rodrigues Barbosa, 2017, p. 21, traducción propia).

En este sentido, los profesionales de la información han ocupado un rol de gran relevancia, al desarrollar pautas y estándares para la evaluación de información. Se han establecido diversas características de las fuentes de información que deben ser tenidas en cuenta para determinar la calidad y confiabilidad de la información, tales como: autoría, alcance de contenido, rigurosidad, objetividad, actualización, presentación y accesibilidad.

Sobre esto, la Unesco (2016) considera imprescindible la formación de la ciudadanía en la alfabetización mediática e informacional, en orden de garantizar su desarrollo en la sociedad. Así es que coloca la evaluación de la información como una herramienta fundamental para el pleno desenvolvimiento de los individuos en las sociedades democráticas.

La gestión y el consumo de información poseen un rol central en la actualidad es por esto que, el papel del profesional de la información, y en particular el de la evaluación de información, se han visto redefinidos. Estos profesionales deben generar herramientas para la evaluación de información que sean de fácil aplicabilidad para todos los individuos, puesto que deben emplearla cotidianamente. Deben tomarse en consideración las críticas que se realizan a los diferentes modelos y listas de criterios de evaluación, ya que se ha señalado que son complejos, contraproducentes y contra intuitivos para la utilización de las personas, lo que se transforma en un elemento desalentador. Se necesitan herramientas de evaluación de información que sean intuitivas y permeables a la hora de emplearlas (Mandalios, 2013).

$\mathrm{Al}$ respecto, Hjorland $(2011 ; 2012)$ ha considerado que las fuentes de información deben ser funcionales a los usuarios, por lo que es muy difícil establecer a priori si una fuente es buena o mala en sí misma, sino que 
su relevancia está dada con la pregunta que intenta responder. Por esto, es necesario apuntar al desarrollo del pensamiento crítico, así como a generar propuestas alternativas de evaluación de la información.

En definitiva, resulta evidente la relevancia que posee el proceso de evaluación de información y, por consiguiente, la selección de información de calidad, en el manejo de información.

\section{ALFABETIZACIÓN EN INFORMACIÓN, COMPETENCIAS EN INFORMACIÓN Y FAKE NEWS}

Hasta aquí se han presentado y analizado la búsqueda y la evaluación de información como dos elementos indispensables en la de adquisición de competencias en información y en el proceso de alfabetización en información. En el presente apartado, se introduce y define el concepto de fake news como tercera categoría analítica y se reflexiona sobre su vínculo con los procesos antes mencionados.

Ahora bien, la búsqueda y evaluación de información adquieren una mayor ponderación en la actualidad, en donde se recurre cada vez más a la web y a las redes sociales como fuentes de información a consultar para informarse. Esto vuelve mucho más complejo el relacionamiento entre los individuos y la información, ya que estas redes han abierto la posibilidad de difundir grandes cantidades de información de forma instantánea. Los viejos canales de producción y difusión de la información se han transformado y ahora todos los usuarios poseen la capacidad de generar, producir y difundir información. Es en este contexto en donde surgen y se propagan las fake news (Alonso-Arévalo y Martín Castilla, 2019; López-Borrull, Vives-Gràcia y Badell, 2018).

Sobre su alcance, se las comprende como:

toda aquella información fabricada y publicada deliberadamente para engañar e inducir a terceros a creer falsedades o poner en duda hechos verificables. Esta definición debería permitirnos diferenciar más fácilmente el periodismo de la propaganda, de los "hechos alternativos" y de las mentiras malévolas (White, 2017, p. 10).

El contacto de los individuos con las fake news aumenta constantemente y tiene lugar cada vez que realizan una búsqueda de información o consultan sus redes sociales. En consecuencia, el desarrollo de las competencias en la búsqueda y evaluación de la información les permite hacer frente al nuevo contexto, en donde se busca inducir al error y desinformar a partir de información falsa o alterada para lograr ciertos objetivos.

Es de suma importancia que los profesionales de la información formen a los individuos en la utilización de las fuentes de información y en qué elementos deben tener presentes para confiar en ellas y validarlas, sobre la base de que la gran mayoría de los individuos está presente y utiliza las redes sociales para informarse. Al respecto de esto, se puede mencionar que:

El término noticias falsas, también conocido como desinformación o hechos alternativos, se utiliza con mayor frecuencia para describir historias completamente inventadas, pero también puede aplicarse a un continuo más amplio de noticias. La evaluación de la calidad de la fuente de información es crucial y es responsabilidad de cada individuo evaluar la información y garantizar que la información sea confiable y veraz. (Courtney, 2017, p. 11, traducción propia)

Si bien la difusión de fake news o la desinformación por parte de los medios de comunicación no son nuevos y siempre se vincularon con la ética en la comunicación y en el periodismo, sí es novedosa la forma en que se viralizan y son compartidas en las redes sociales. Recientemente Vosoughi, Roy y Aral (2018), luego de analizar diversos rumores compartidos en Twitter concluyeron que las fake news se propagan mucho más rápido y más lejos que las noticias verdaderas. Algunas de las explicaciones que encuentran es que estas, por lo general, tratan sobre novedades que suelen repercutir en mayor grado en los usuarios. A su vez, consideran que la propagación se debe principalmente a que los usuarios comparten este tipo de información y no tanto a la existencia de bot que se encarguen de difundir esta información.

En este sentido, Budd y Stewart (2018) consideran que las nuevas plataformas y redes sociales, como espacios en donde se diseminan la información y las fake news, han logrado evitar los tradicionales métodos 
utilizados en los procesos de alfabetización en información a la hora de evaluar las fuentes de información, ya que poder identificar aquellas que sean autorizadas y confiables es cada vez más complejo. A su vez, reparan en que aquellos individuos que no poseen la capacidad de analizar críticamente la información tienen mayores probabilidades de caer en noticias falsas o malintencionadas.

La rapidez y sencillez con que se difunden las fake news hace indispensable que se implementen nuevos mecanismos de filtrado, evaluación y control de la información, tanto por el usuario como por los profesionales de la información. Para Morales Campos (2018), es necesario que los profesionales de la información brinden nuevos tipos de servicios, como por ejemplo la verificación de datos, la comparación y acreditación de fuentes, así como acceso a servicios de filtrado, que permitan bloquear este tipo de información.

En consideración a lo aportado por Budd y Stewart (2018), Petrucco y Ferranti (2017) añaden que, el desarrollo de las habilidades digitales, en particular las de buscar y evaluar información, son competencias fundamentales para desenvolverse correctamente en internet. Así es que, una forma adecuada de incentivar estas competencias es formar y desarrollar el pensamiento crítico sobre las fuentes de información. Si son capaces de incorporar el pensamiento crítico a los procesos de búsqueda y evaluación estarán parados en un mejor escenario para enfrentar las fake news.

Así como se mencionó anteriormente, frente al crecimiento de la difusión de las fake news, se torna indispensable que los individuos desarrollen competencias de evaluación de la información, sobre todo en el entendido de que estas circulan con mayor rapidez y poseen mayor alcance que el resto. Tanto el documento de la Unesco (2016) como Ireton, Posetti (2018) coinciden con que el desarrollo de la alfabetización mediática e informacional cobra cada vez más importancia al posicionarse como una herramienta que combate la información falsa y la desinformación. Asimismo, recalca que es fundamental desarrollar el pensamiento crítico como instrumento a la hora de enfrentar la difusión de las fake news.

Sobre esta misma línea, la nueva definición del CILIP (2018) sobre alfabetización en información repara en que se trata de un conjunto de habilidades y competencias que todos los individuos necesitan para realizar tareas con la información. A su vez, estima que es necesario incorporar el pensamiento crítico, así como la comprensión ética del uso de la información con respecto a sus implicancias políticas.

Para López-Borrull, Vives-Gràcia y Badell (2018) el entorno de las fake news representa una nueva oportunidad para el trabajo de los profesionales de la información. Un elemento de gran trascendencia es que, la alfabetización en información ocupe un rol central en la formación de los individuos, que les permita diferenciar las fuentes de información fiables e identificar la información falsa de la que no lo es.

Para la IFLA (2018a; 2018b), los profesionales de la información y las bibliotecas poseen un gran compromiso institucional y ético para que los usuarios accedan a información confiable, ya que ofrecen las herramientas y destrezas para detectarla y reconocerla. A su vez, colaboran con los ciudadanos en la evaluación crítica de la información en sus diversos soportes. La IFLA ${ }^{3}$ subraya que es indispensable el desarrollo de la alfabetización en información para hacer frente al contexto actual, así como, para el buen funcionamiento de la sociedad.

La búsqueda y evaluación de la información se presentan como herramientas imprescindibles para todos los individuos a la hora de desarrollarse en la actual sociedad de la información. Sumado a esto, el desarrollo del pensamiento crítico frente al mundo informacional también es un componente clave. Si bien los profesionales de la información por muchos años han contribuido a la búsqueda y la evaluación de las diversas fuentes de información, el desarrollo del entorno web y de las redes sociales hace indispensable que muchas de estas herramientas se adapten a la nueva realidad y sean instrumentos apropiados para que los individuos sean capaces de manejar y usar apropiadamente la información. 


\section{Conclusiones}

En el contexto actual de la sociedad de la información y de propagación de fake news, el manejo de la información se torna indispensable para el desarrollo individual y colectivo. La búsqueda y la evaluación de información son dos pilares fundamentales del manejo de información, por lo que es necesario formar a los sujetos en este sentido. También son componentes centrales para enfrentar la desinformación que resulta del acceso a información falsa y fake news.

Así, las competencias en información, en cuanto a la capacidad de búsqueda, acceso, evaluación, selección y uso de la información, son centrales en el manejo de la información y la satisfacción de las necesidades informacionales de los individuos para el desarrollo de su vida cotidiana. Por lo que, fomentar su desarrollo es primordial para la generación de ciudadanos críticos y capaces de desenvolverse en la sociedad.

En este escenario, la figura del profesional de la información toma relevancia al poseer la formación adecuada para insertarse y liderar los procesos de adquisición de competencias en el manejo, uso y comunicación de la información por parte de los ciudadanos. Sin embargo, es importante remarcar que, los procesos de adquisición de competencias en el manejo de información no son exclusivos del área de la ciencia de la información ni se remiten únicamente a las unidades de información, sino que son procesos transversales al desarrollo y formación de los individuos.

Es necesario que, en el proceso de alfabetización y adquisición de competencias en información, se desarrollen capacidades críticas para la búsqueda y la evaluación ya que, son dos componentes de impacto significativo para detectar las fake news.

Estas, como mecanismo de desinformación de la sociedad, no son un elemento nuevo. Sin embargo, en la actualidad, el acceso masivo a Internet y a las redes sociales ha generado un escenario de diseminación continua de este tipo de noticias. Si bien este fenómeno, ha sido abordado en diversas ocasiones desde el campo de la comunicación, en relación a la ética de la comunicación y del periodismo, debe entenderse como un fenómeno de doble arista: informacional y comunicacional. Ambos lados se encuentran estrechamente relacionados y deben ser analizados y trabajados de manera dialógica para combatir este problema.

Para concluir, los individuos deben tomar conciencia de que este tipo de información se difunde con el objetivo de engañar y desinformar, y que estos objetivos poseen consecuencias sociales, culturales y políticas. Esto, posiciona al profesional de la información en un lugar de gran responsabilidad como actor que debe aportar al tratamiento de las competencias en información. Es importante el desarrollo de estas competencias para diferenciar la información que refiere a hechos reales de aquella que es falsa, así como para fomentar una comunicación ética de la información, desalentando la diseminación de fake news.

\section{REFERENCIAS}

Alonso-Arévalo, J., y Martín Castilla, S. (2019). El papel de las bibliotecas en un mundo de noticias falsas. Desiderata, 11(1). Recuperado de https://dialnet.unirioja.es/servlet/articulo?codigo $=6885101$

American Association of School Librarians \& Association for Educational Communications and Technology (1998). Information power: building partnerships for learning. Chicago: ALA. Recuperado de https://www.ala.org/ala/a asl/aaslproftools/informationpower/InformationLiteracyStandards_final.pdf

Argudo, S., y Pons, A. (2012). Mejorar las búsquedas de información. Barcelona: UOC.

Batista Rojas, O.; Hernández Rodríguez, Y.; Hernández López, X. Y. y González Cedeño, J. de M. (2011). Caracterización de competencias informacionales en estudiantes del Policlínico Docente Meneses. 2010. Revista cubana de informática médica, 3(2), 100-112. Recuperado de http://scielo.sld.cu/scielo.php?script=sci_arttext \&pid=S1684-18592011000200002

Budd, J. M. y Lloyd, A. (2014). Theoretical foundations for information literacy: a plan for action. Asistઐ t, 51(1). Recuperado de https://asistdl.onlinelibrary.wiley.com/doi/epdf/10.1002/meet.2014.14505101001 
Budd, J. M., y Stewart, K. N. (2018). News, fake news, and critical authority. En S. Kurbanoğlu, J. Boustany, S. Špiranec, E. Grassian, D. Mizrachi, y L. Roy (Eds.), Information literacy in the workplace, 810, 227-232.

CAUL Council of Australian University Librarians (2002). Normas sobre alfabetización en información. Boletín de la Asociación Andaluza de Bibliotecarios, 68. Recuperado de http://eprints.rclis.org/5944/1/68a4.pdf

Ceretta Soria, M. G.; Canzani Cuello, J., y Cabrera Castiglioni, M. (2016). Competencias lectoras y competencias en información: espacios de convergencia. Ciência da informação, 45(2). Recuperado de http://revista.ibict.br/cii nf/article/view/3802/3356

Ceretta Soria, M. G., y Gascue, A. (coord.) (2015). Modelo Pindó: un modelo de alfabetización en información para el Plan Ceibal. Montevideo: Ediciones Universitarias. Recuperado de https://www.colibri.udelar.edu.uy/jspui/bi tstream/20.500.12008/9027/1/ceretta_modelo_pindo.pdf

Cid Leal, P., y Perpinya Morera, R. (2013). Cómo y dónde buscar fuentes de información. Bellaterra: Universitat Autònoma de Barcelona.

CILIP (2018). CILIP Definition of information literacy. London: Information literacy group. Recuperado de https://cdn.ymaws.com/www.cilip.org.uk/resource/resmgr/cilip/information_professional_and_news/pre ss_releases/2018_03_information_lit_definition/cilip_definition_doc_final_f.pdf

Çoklar, A. N.; Yaman, N. D. y Yurdakul, I. K. (2017). Information literacy and digital nativity as determinants of online information search strategies. Computers in human behavior, 70, 1-9. Recuperado de https://doi.org/10 $.1016 /$ j.chb.2016.12.050

Corominas, J. (2012). Breve diccionario etimológico de la lengua castellana. Madrid: Gredos.

Courtney, I. (2017). In an era of fake news, information literacy has a role to play in journalism education in Ireland: an exploratory study of journalism and media faculties and the interaction with their academic libraries. Master thesis. Information and Library Management Dublin Business School, Dublin. Recuperado de https://esource. dbs.ie/bitstream/handle/10788/3303/msc_courtney_i_2017.pdf?sequence=1\&isAllowed=y

Cruz Gil, M. del C. (2015). Modelos de búsqueda y recuperación de la información. Somonte-Cenero, Gijón, Asturias: Trea.

Delis Alfonso, L. M. (2016). Evaluación de fuentes de información en taller de habilidades informacionales. Bibliotecas. Anales de investigación, 12(12). Recuperado de https://dialnet.unirioja.es/servlet/articulo?codigo= 5704487

Eduteka (2007). Modelo Gavilán 2.0: Una propuesta para el desarrollo de la competencia para manejar la información (cmi). Eduteka. Recuperado de http://www.eduteka.org/pdfdir/ModeloGavilan.pdf

Elisondo, R. C. y Donolo, D. S. (2014). Creatividad y alfabetización informacional. El desafío en cuatro propuestas. Panorama, 8(15). Recuperado de http://journal.poligran.edu.co/index.php/panorama/article/view/547/485

Fernández-Ramos, A. (2014). El papel de las bibliotecas en el acceso a recursos web de calidad. Perspectivas em ciência da informação, 19(1). Recuperado de http://portaldeperiodicos.eci.ufmg.br/index.php/pci/article/view/1811/ 1248

García Rodríguez, R. (2017). La teoría de la actividad en el estudio del comportamiento informacional humano: consideraciones fundamentales. Informação em pauta, 2(1), 50-72. Recuperado de http://www.periodicos.ufc.b r/informacaoempauta/article/view/6549/30024

Giffoni Dutra, F., y Rodrigues Barbosa, R. (2017). Modelos e critérios para avaliação da qualidade de fontes de informação: uma revisão sistemática de literatura. Informação \& sociedade: estudos, 27(2). Recuperado de https ://periodicos.ufpb.br/ojs2/index.php/ies/article/view/32676

Hjorland, B. (2011). Evaluation of an information source illustrated by a case study: effect of screening for breast cancer. Journal of the American Society for Information Science and Technology, 62(10).

Hjorland, B. (2012). Methods for evaluating information sources: an annotated catalogue. Journal of information science, $38(3)$.

IFLA (2018a). Declaración de la IFLA sobre las noticias falsas. Recuperado de https://www.ifla.org/files/assets/faife /statements/ifla-statement-on-fake-news-es.pdf 
IFLA (2018b). Respuesta de la IFLA a las Noticias Falsas: desarrollar destrezas y luchar contra la censura. 25 Agosto 2018 From the Annual Conference. Recuperado de https://www.ifla.org/ES/node/67336

Ireton, C., y Posetti, J. (2018). Journalism, fake news \& disinformation: handbook for journalism education and training. Paris: Unesco. Recuperado de http://unesdoc.unesco.org/images/0026/002655/265552E.pdf

Ladrón de Guevara, M. C. (2017). Estudio e investigación: buscar, encontrar y usar información. Córdoba: Brujas; Universidad Nacional de Córdoba.

Lau, J. (2007). Directrices sobre Desarrollo de Habilidades Informativas para el Aprendizaje Permanente. IFLA. Recuperado de https://www.ifla.org/files/assets/information-literacy/publications/ifla-guidelines-es.pdf

Lopez-Borrull, A.; Vives-Gràcia, J. y Badell, J.-I. (2018). Fake news, ¿̇amenaza u oportunidad para los profesionales de la información y la documentación? El profesional de la información, 27(6), 1346. Recuperado de https://doi.o $\mathrm{rg} / 10.3145 /$ epi.2018.nov.17

López Flamarique, M. (2017). Buscar, evaluar y seleccionar información digital: una experiencia didáctica en Educación Secundaria Obligatoria. Tesis doctoral. Mondragon Unibertsitatea, Mondragón (País Vasco). Recuperada de http://academica-e.unavarra.es/xmlui/handle/2454/26022

Mandalios, J. (2013). RADAR: an approach for helping students evaluate Internet sources. Journal of information science, 39(4).

Mata, M. L. da, Silva Casarin, H de C. y Marzal, M. A. (2016). Da educação de usuários à competência em informação: perspectivas conceituais. En: Melo Alves, F. M., Delfini Correa, E. C., y Oliveira Lucas, E. R. de (Orgs.). Competência em informação: políticas públicas, teoria e prática. Salvador - Bahía: EDUFBA. Recuperado de h ttps://repositorio.ufba.br/ri/handle/ri/22598

Morales Campos, E. (2018). Desinformación en la sociedad de la información y el conocimiento. En Morales Campos, E. (coord.) (2018). La posverdad y las noticias falsas: el uso ético de la información. México: UNAM, Instituto de Investigaciones Bibliotecológicas y de la Información. Recuperado de http://ru.iibi.unam.mx/jspui/bitstream/ IIBI_UNAM/L151/2/posverdad_noticias_falsas_s.pdf

Núñez Paula, I. A. y Zayas Caballero, I. (2016). Análisis de modelos sobre comportamiento informacional, desde un enfoque socio-psicológico. Bibliotecas. Anales de investigación, 12(1), 63-89. Recuperado de https://dialnet.un irioja.es/servlet/articulo?codigo $=5704490$

Odede, I., y Zawedde, N. (2018). Information literacy skills in using electronic information resources. Library philosophy and practice (e-journal), 1947. Recuperado de http://digitalcommons.unl.edu/libphilprac/1947

Petrucco, C., y Ferranti, C. (2017). Developing critical thinking in online search. Journal of E-learning and knowledge society, 13(3). Recuperado de https://doi.org/10.20368/1971-8829/1390

Piwowarski, B., y Blanco González, R. (2011). Introducción a la recuperación de la información. En Cacheda Seijo, F.; Ferna\#ndez Luna, J. M. y Huete Guadix, J. F. (2011). Recuperacio\#nde informacio\#n: un enfoque pra\#ctico y multidisciplinar. Madrid: RA-MA.

Ruiz, M. E. P. y Chapman, M. C. S. (2017). Las competencias informacionales en las ciencias médicas. Revista de la Facultad de Ciencias Médicas de la Universidad de Guayaquil, Número Especial de Pedagogía. Recuperado de h ttp://eluniversitario.edu.ec/revistas/index.php/RFCM/article/view/221

Salvador Oliván, J. A. y Angós Ullate, J. M. (2001) ¿Evaluar la calidad de los recursos Web o simplemente filtrarlos? Documentación de las ciencias de la información, 24. Recuperado de http://revistas.ucm.es/index.php/DCIN/a rticle/view/DCIN0101110105A/19513

Unesco (2016). Marco de avaliação global da alfabetização midiática e informacional: disposição e competências do país. Brasilia: Unesco. Recuperado de https://unesdoc.unesco.org/ark:/48223/pf0000246398

Vosoughi, S., Roy, D., y Aral, S. (2018). The spread of true and false news online. Science, 59(6380), 1146-1151. Recuperado de https://doi.org/10.1126/science.aap9559

Voutssas-Márquez, J. (2017). Confianza e información digital: Bibliotecas archivos y web. México: UNAM, Instituto de Investigaciones Bibliotecológicas y de la Información. Recuperado de http://iibi.unam.mx/voutssasmt/docu mentos/confianza_informacion_digital.pdf 
White, A. (2017). Información falsa. La opinión de los periodistas. Correo de la Unesco, 2. Recuperado de https://un esdoc.unesco.org/ark:/48223/pf0000252318_spa

\section{Notas}

1 Cabe recordar que varias normas y modelos de alfabetización en información consideran que los procesos de búsqueda y evaluación de fuentes de información son indispensables y son las primeras etapas para satisfacer una demanda de información. Para ver algunas de estas normas y modelos, se puede consultar: CAUL (2002); Ceretta Soria y Gascue (2015); Eduteka (2007); Lau (2007).

2 En el marco de la ciencia de la información se han desarrollado varios modelos que comprenden la búsqueda y el comportamiento informacional de los individuos. Aquí no se busca abordar esta temática, sino indagar en las competencias de búsqueda y evaluación de información para el desenvolvimiento de los individuos en la sociedad. Si se quiere aproximar a estos temas, se puede ver: Cruz Gil (2015); García Rodríguez (2017); Núñez Paula, Zayas Caballero (2016).

3 La IFLA en 2017 publicó recomendaciones de Cómo las bibliotecas pueden ayudar a conseguir soluciones reales para las noticias falsas junto con una infografía titulada Cómo detectar noticias falsas, que permite contar con algunos elementos y herramientas a la hora de detectar las fake news. La infografía se hizo viral y fue traducida a 37 idiomas. Se puede consultar en: https://www.ifla.org/ES/node/11631 